\title{
Simulación y Juegos de rol como herramienta de innovación en la asignatura Deontología y Profesionalismo
}

\author{
MariLuz Gordo ${ }^{\mathrm{a}}$, Marta Fernández-Diego ${ }^{\mathrm{a}}$, Faustino Alarcón ${ }^{\mathrm{a}}$, Andrés Boza ${ }^{\mathrm{a}}$ \\ ${ }^{a}$ Departamento de Organización de Empresas. Universitat Politècnica de València. \\ Equipo de Innovación Educativa AEGECATE. (magormon@upvnet.upv.es, \\ marferdi@omp.upv.es, faualva@omp.upv.es, aboza@omp.upv ).
}

\begin{abstract}
Simulation and role-playing are techniques that allow working the competence Innovation, Creativity and Entrepreneurship. This article presents the design and development of an activity based on simulation and role play totally located in the future professional field of our students, Computer Engineering. It should be highlight, the high degree of participation of the students in the activity carried out and their involvement in the role that they have to assume.
\end{abstract}

Keywords: Simulation, Role Playing, Competition, Innovation, Creativity, Entrepreneurship.

\begin{abstract}
Resumen
La simulación y los juegos de rol son técnicas que permiten trabajar la competencia Innovación, Creatividad y Emprendimiento. En este artículo se presenta el diseño y desarrollo de una actividad basada en la simulación y los juegos de rol, totalmente ubicado en el futuro ámbito profesional de nuestros estudiantes, la Ingeniería Informática. Cabe destacar, el alto grado de participación del alumnado en la actividad realizada y su implicación dentro del rol que se le propone asumir.
\end{abstract}

Palabras clave: Simulación, Juego de Rol, Competencia, Innovación, Creatividad, Emprendimiento.

\section{Introducción}

La innovación, creatividad y emprendimiento ha sido un tema de considerable interés en la educación desde hace algún tiempo, y una de las competencias más demandadas por las empresas. Promover la adquisición de esta competencia es uno de los aspectos a resolver. La innovación, la creatividad y el espíritu empresarial son un saber hacer complejo que se desarrollará en la educación superior. Este complejo conocimiento debe especificarse en el diseño de nuevos currículos a través del conjunto de competencias específicas y transversales que los estudiantes deben desarrollar.

La simulación y juegos de rol son técnicas que permiten trabajar esta competencia.

De acuerdo con lo establecido en el artículo de García et al. (2011) dentro de las metodologías activas que fomentan la innovación, son ampliamente empleadas el trabajo en parejas, lluvia 
Simulación y Juegos de rol como herramienta de innovación en la asignatura Deontología y Profesionalismo

de ideas, rueda de ideas, debates, grupos de discusión, etc. Si bien estos autores indican lo siguente sobre la técnica de la simulación:

Además de estas técnicas, se encuentra el uso de la simulación en el proceso de enseñanza aprendizaje, que, según Ruben (1999), basándose en los enfoques de aprendizaje de finales de los años 60, es una metodología que supera las limitaciones de la enseñanza tradicional. De hecho, y de acuerdo con este autor, el uso de las simulaciones como metodología educativa conlleva una mejora en el proceso de aprendizaje del propio estudiante, facilita la interactividad entre los estudiantes, el trabajo colaborativo y, en definitiva, un aprendizaje más activo.

También en dicho artículo se define juego de rol (rol play o rol playing) como:

Técnica en la que cada participante desempeña un papel concreto en el contexto de una situación simulada siguiendo determinadas reglas y en la que interactúa con otros participantes del mismo juego de simulación.

La aplicabilidad de los juegos de rol tambien la encontramos en el trabajo de Perea (2008) :

Por ejemplo, el hecho de que un jugador de rol diseñe creativamente un personaje con un conjunto de caracteristicas morales especificas, le permite experimentar y explorar principios de acción y criterios de estilo de existencia, en su relación consigo mismo y con otros, en el juego estratégico entre las libertades que intervienen.

Y ligándolo con la competencia de Innovación, emprendimiento y creatividad, viene al hilo la mención a los autores Jiménez y Moncholi (2009) :

La sociedad ya ha tomado conciencia de que la creatividad es un derecho y una exigencia social; esta creatividad, indispensable en muchas disciplinas, puede desarrollarse a través de la técnica de juegos de rol, con la que, además, se potencia el aprendizaje y se suscita la curiosidad de los jugadores. Los juegos de rol, a pesar de que los personajes y la situación que se simula vienen definidos a priori, atendiendo a determinadas reglas impuestas por un director (normalmente el profesor o grupo de profesores que imparten una determinada materia), no exigen un diálogo concreto a los jugadores, siendo éstos libres para poder reconducir la situación o problemática planteada en aras de encontrar ciertas soluciones.

Por ello se puede concluir que estas técnicas abren una ventana a la imaginación y la creatividad de forma muy espontánea y natual, y por tanto facilitan el desarrollo de la competencia Creatividad, Innovación y Emprendimiento.

La innovación docente sobre la competencia de Creatividad, Innovación y Emprendimiento se desarrolla en el marco de la asignatura Deontología y Profesionalismo.

Esta asignatura se encuentra en el segundo año del Grado de Ingeniería Informática de la Escuela Tècnica Superior de Ingeniería Informática y se divide en tres bloques: "Profesionalismo", "Aspectos legales" y "Ética". 
- Profesionalismo

La asignatura comienza con el estudio de la "profesión" en general para, rápidamente, particularizar en la profesión informática. El tema pasa sobre la historia de la informática y analiza cuándo la informática comenzó a ser consciente de su existencia, desvinculada de otras profesiones.

- Aspectos jurídicos

En esta parte del curso, la asignatura da una idea de las principales facetas de esta profesión sujetas a la ley, teniendo en cuenta las principales normas. Obviamente, es imposible abordar con plenitud cada ley, regulación y otros preceptos legales que afectan a la profesión. Por lo tanto, el tema centra la atención en aquellas áreas que reciben más interés, tanto por la atención prestada por los tribunales, como por el impacto en la sociedad civil. Así, la asignatura trata de cubrir al menos el marco legal básico para la buena práctica profesional.

- Deontología y ética

El tema introduce la ética y su relación con la empresa y, sobre todo, con la profesión informática. La razón de su inclusión es elemental: si es cierto que la ley marca algunos canales de acción, la última barrera, que nos marca las delgadas líneas rojas que no deben cruzarse, se llama ética. Como es un tema inusual para una carrera en tecnología, comienza con el establecimiento de algunos conceptos básicos, para luego discutir la ética empresarial sin olvidar la ética individual o la ética de la sociedad en general. El tema introduce cuestiones clave como el equilibrio social o los códigos de ética y, por supuesto, pone especial énfasis en la relación entre la ética y la ciencia de la computación.

\section{Objetivos}

El objetivo del trabajo es presentar el diseño y desarrollo de una actividad basada en simulación y juego de rol, llevada a cabo en la asignastura Deontología y Profesionalismo para la adquisición de la competencia transversal creatividad, innovación y emprendimiento. La actividad han sido creada íntegramente por los profesores basándose en la experiencia en la docencia de la asignatura.

\section{Desarrollo de la Innovación}

La actividad propuesta se basa en simular situaciones en contextos determinados en las que los alumnos deben ubicarse. En esta actividad, denominada "Fuga de Datos del Hospital de Fuenlabrada", los alumnos simulan estar en un determinado puesto de trabajo y formar parte de un proceso de investigación. Se introduce la simulación recreando en el aula el contexto de un hospital, y más concretamente el departamento de informática de este.

La base de la simulación está en presentar situaciones reales (o realistas), y en el caso de la actividad "Fuga de datos" la actividad toma su origen en la existencia de una noticia publicada en prensa sobre la difusión de datos de carácter personal por parte del hospital incurriendo en una infracción legal. Se escogió esta situación por considerarse que facilita al alumno la conexión con la realidad, la aplicabilidad de los conceptos trabajados y el conocimiento de problemas contemporáneos. 
Simulación y Juegos de rol como herramienta de innovación en la asignatura Deontología y

\subsection{Contexto}

Como se indicó anteriormente, la actividad se desarrolla en la asignatura Deontología y Profesionalismo, asignatura cuatrimestral obligatoria de segundo curso del Grado de Ingeniería Informática, y tiene un tamaño medio de alumnos por grupo: de 20 a 50 alumnos.

\subsection{Desarrollo}

La actividad pretende, de una forma práctica y amena, reforzar la aplicación de la normativa española de protección de datos. Asimismo se plantean varios dilemas morales para provocar la reflexión de los alumnos. La actividad se realiza en parejas.

La actividad se divide en varias partes:

- PARTE I (10 minutos)

\section{LECTURA DE NOTICIA}

"Hospital de Fuenlabrada releva a dos cargos por la filtración datos" 11/04/2014 El País http://ccaa.elpais.com/ccaa/2014/09/11/madrid/1410432868_373337.html

- $\quad$ PARTE II (20 minutos):

JUEGO DE ROL Preparación entrevista Trabajo en equipos de 4 personas: Dos alumnos adoptarán el rol de informáticos del hospital y los otros dos investigadores. Se presenta a continuación parte del enunciado que se da a los alumnos:

\section{Alumnos informáticos del Hospital:}

Trabajáis como responsables de informática en el hospital de Fuenlabrada cuando ha tenido lugar el suceso que reporta la noticia. Os han informado de que van a abrir una investigación y que posiblemente alguien quiera entrevistarse con vosotros. Ante ello conviene que os preparéis bien la información que os pueden solicitar. Tenéis que trazar un argumento sólido.

Algunas preguntas que podéis plantearos (y cuyas respuestas deberéis plasmar en el documento)

1) ¿Qué tipo de datos manejáis? ¿A qué nivel de seguridad corresponden?

2) ¿Quién es el responsable del fichero y el encargado del tratamiento?

3) ¿Tenéis documento de seguridad? ¿Lo empleáis habitualmente?

4) ¿Qué sistemas de seguridad tenéis diseñado en vuestro sistema?

5) ¿Quién os ordenó realizar la cesión de datos?

6) ¿Cómo se desarrolló esta actuación?

(c) EY-NC-ND 2017, Universitat Politècnica de València 


\section{Alumnos investigadores:}

Sois los informáticos encargados de llevar a cabo la investigación sobre lo ocurrido en el Hospital de Fuenlabrada. Os entrevistaréis con los responsables de informática del hospital. Preparar las preguntas que les planteariais con el fin de valorar las responsabilidades y determinar si ha habido una actuación inadecuada argumentándolo con la ley en la mano. Este caso ha tenido mucha repercusión mediática y se requiere actuar con profesionalidad y con rigor. ;Se buscan responsables!

Elementos a considerar:

1) Preguntas a plantear (Las elaboran los investigadores a modo de borrador para la entrevista)

2) Artículos de la legislación (Por ejemplo Ley Orgánica 15/1999 de Protección de Datos de Carácter Personal, RD 1720/2007 por el que se aprueba el Reglamento de desarrollo de la Ley Orgánica 15/1999 de protección de datos de carácter personal, etc.) a tener en consideración por ser aplicables a este caso.

3) Y si, además de hablar de datos, nos encontráramos con los siguientes casos, valora los posibles delitos cometidos. Indica legislación y clasificación del delito según el convenio de ciberdelincuencia, en su caso:

- Se ceden los datos junto con la aplicación que los contiene

- Se ceden los datos a cambio de dinero

- $\quad$ PARTE III (20 minutos):

JUEGO DE ROL Realización entrevista. Los alumnos con el rol "informático" se unen con los del rol "investigador" llevando a cabo una entrevista para obtener la información necesaria. Se presenta a continuación parte del enunciado que se da a los alumnos:

1) Resume lo acontecido durante el interrogatorio

2) Valora a tus compañeros con la información proporcionada

En el caso de los investigadores, además:

3) Determinar qué tipo de responsabilidad se deriva

4) Si consideráis que se ha cometido algún delito clasificarlo e indicar el artículo concreto y normativa incumplidos.

- $\quad$ PARTE IV (15 minutos):

DILEMA MORAL en EQUIPO. Todos los alumnos asumen el rol de informático para introducir dos situaciones nuevas. Se presenta a continuación parte del enunciado que se da a los alumnos: 
Pongámonos en situación: Ahora somos todos informáticos del departamento de informática en el Hospital de Fuenlabrada.

1) Observamos que se está realizando la cesión de datos sin tener seguridad de que se cumplen todas las garantías que exige la ley, por ejemplo no se está pidiendo autorización a los interesados. ¿Qué haríamos?

2) Observas que uno de los pacientes afectados es un familiar tuyo. Si dices algo a tus superiores sobre los incumplimientos es posible que se paralice el proceso y se demore la operación de tu tío. ¿Cómo actuarías?

\section{Resultados}

\subsection{Implicación del alumnado en la actividad:}

Cuando se introduce la actividad, se distingen dos tipos de reacciones entre el alumnado, los "favorables" a este tipo de actividades más dinámicas y animadas, y los "reacios" que, al salirse de las actividades típicas o tradiconales, pueden encontrar dificultades o que por tener que teatralizar les supone un esfuerzo mayor que una actividad convencional.

La experiencia como docente en la aplicación de juegos de rol es que, al finalizar la actividad,se unifican las reacciones y los alumnos, en general, muestran entusiasmo. La realidad es que se ponen en la piel de otros empatizando con los personajes, elucubran argumentos con inmediatez y se divierten, a la vez que asimilan conceptos. Por tanto, resumiendo, es de destacar el alto grado de participación del alumnado en la actividad realizada y su implicación dentro del rol que debe asumir en la actividad.

\subsection{Implicaciones para el profesorado:}

Se tratade una actividad que requiere de gran implicación del profesor, ya que debe ser totalmente guiada por varias razones: Es una actividad que se sale de los estándares habituales, hay que conseguir una contextualización lo más fidedigna posible de la situación - dentro de las limitaciones existentes-, y puesto que es muy participativa, se debe asegurar que se mantenga el orden y unas cuotas no excesivas de sonoridad en la sala.

\subsection{Evaluación}

El resultado de la actividad en lo que se refiere a participación se maximiza al trabajar en grupos pequeños.Con ello se consigue, además, que los alumnos actúen de forma totalmente espontánea.

En cuanto a notas, las puntuaciones de los alumnos valoradas por el profesor son bastante buenas. La práctica se dio en 2 grupos, uno de 14 alumnos y otro de 10. Las notas oscilaron entre 0,2 y 0,25 sobre 0,25 (entre 8 y 10 en base 10), lo cual es un resultado bastante satisfactorio.

Como evidencias, se dispone de los documentos cumplimentados con los alumnos y corregidos por el profesor. 


\subsubsection{Mejoras para futuras evaluaciones}

A posteriori de la realización de la actividad, se consideró interesante introducir el concepto de co-evaluación desde una perspectiva cualitativa para ser utilizada en próximos cursos. Para ello se diseñó un sencillo cuestionario que deberá ser respondido por los alumos. Se trata de que dentro del equipo de cuatro alumnos, evalúen por pares, el rol de los otros, lo cual sumará información y podrá ser incorporada a la nota final, adicionándola a la obtenida por el profesor, aplicando el peso que se considere.

\begin{tabular}{|c|c|c|c|}
\hline \multicolumn{4}{|c|}{$\begin{array}{c}\text { EVALUA.A.TUS.COMPANEROS.SIENDO. } \\
\text { IMPARCIAL.Y.JUSTOA }\end{array}$} \\
\hline$a$ & $\theta r$ & $\Theta r$ & \\
\hline \multicolumn{4}{|l|}{ Contestan-TODOSa } \\
\hline $\begin{array}{l}\text { ¿Las·preguntas/respuestas. } \\
\text { estaban·preparadas?a. }\end{array}$ & $a$ & $a$ & $a$ \\
\hline \multicolumn{4}{|l|}{ a } \\
\hline \multicolumn{4}{|l|}{ Contestan-LOS·INFORMÁTICOSá } \\
\hline $\begin{array}{l}\text { ¿Nos·han'explicado·el·motivo· } \\
\text { de·la·entrevistad }\end{array}$ & $a$ & $a$ & $a$ \\
\hline $\begin{array}{l}\text { ¿Conocían·la·materia·que· } \\
\text { trataban?’a }\end{array}$ & $a$ & $a$ & $a$ \\
\hline \multicolumn{4}{|l|}{ d } \\
\hline \multicolumn{4}{|c|}{ Contestan-LOS·INVESTIGADORESă } \\
\hline $\begin{array}{l}\text { ¿Tenían·los·informáticos·un· } \\
\text { argumento·sólido?ă }\end{array}$ & $a$ & $a$ & $a$ \\
\hline ¿Han·sabido·defenderse?व & $a$ & $a$ & $a$ \\
\hline
\end{tabular}

Figura 1 Co-evaluación entre alumnos

El anterior cuestionario se enfoca hacia la co-evaluación de la participación del alumnado en la actividad. Otra cuestión es la valoración de la actividad en si misma por parte del alumnado. Se ha diseñado una pequeña encuesta para evidenciar la percepción de los alumnos. De esta manera, les solicitamos que sean parte activa de la mejora. Asimismo, las aportaciones facultarán al profesor para realizar adaptaciones de la actividad con el fin de adecuarla lo máximo posibles a las necesidades y expectativas de los alumos, sin sacrificar los contenidos del aprendizaje. 


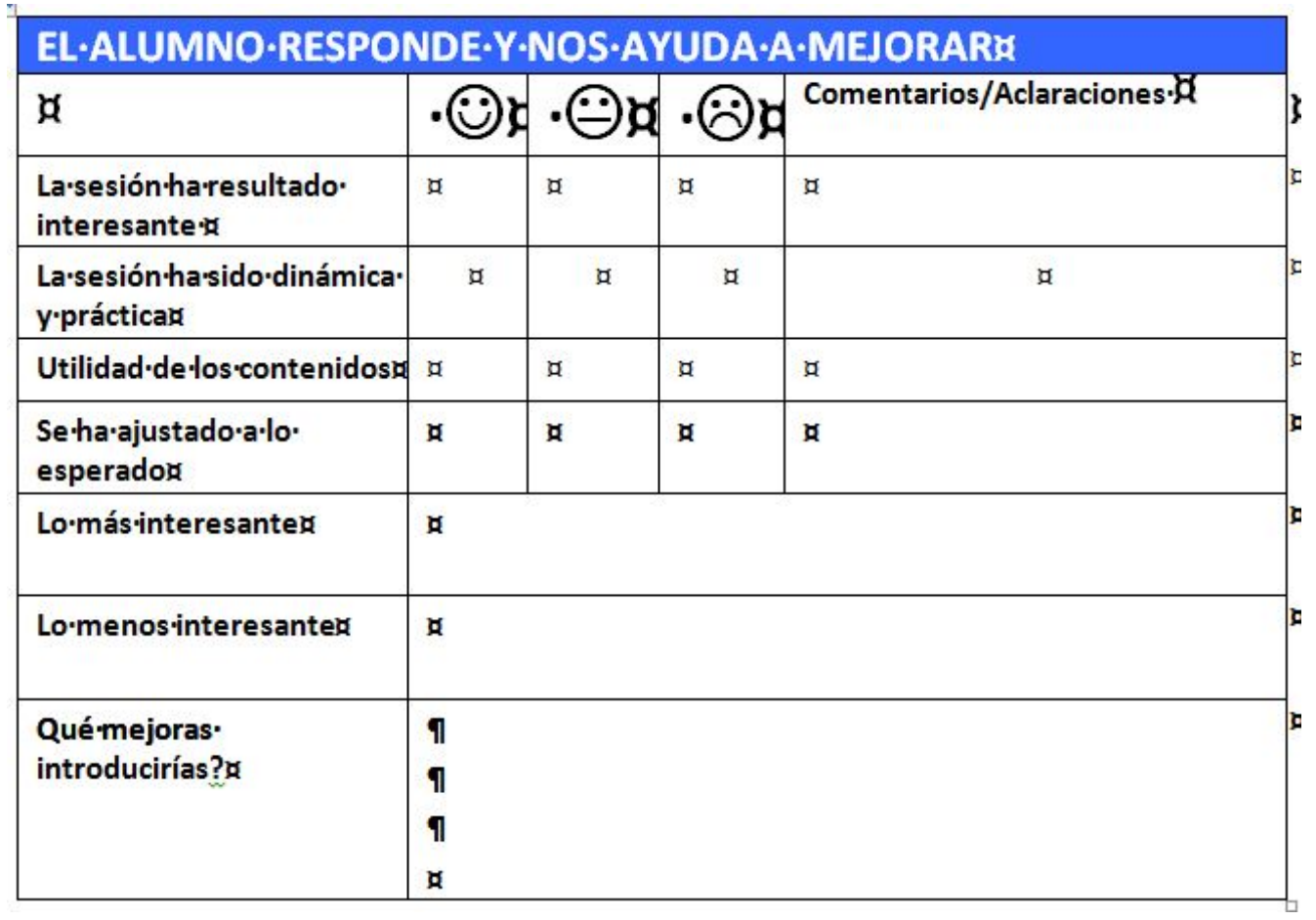

Figura 2 Encuesta de satisfacción planteada

\subsection{Ventajas y dificultades en la aplicación de esta técnica}

El diseño de la actividad vino precedido del análisis de diferentes metodologías como i) la lectura de un texto y posterior reflexión, debate y extracción de conclusiones en el documento de la memoria, o ii) proponer el artículo de prensa que se presenta en la práctica y debatir sobre él. Sin bien, finalmente se consideró más adecado el diseño final planteado en este artículo, presentando las siguientes ventajas:

- La aplicación de la simulación y los juegos de rol permite dinamizar y convertir en algo ameno la aplicación de un tema cuyo enfoque teórico pueda ser tedioso.

- Es extremadamente versátil y permite tratar temas diversos en cuanto a contenido y complejidad.

- Contribuye a crear un alto grado de participación del alumnado en la actividad realizada.

- Se fomenta el trabajo en equipo.

- Con la ayuda del profesor se puede lograr fácilmetne la implicación del alumno en el rol que debe asumir.

- Se desarrolla la creatividad del alumnado al permitir libertad en el enfoque que debe dar cada alumno o grupo de alumnos a su intervención,

- Se fomenta la capacidad de empatizar con otros, al ponerse en su piel (responsables de informática del hospital) y entender la verdadera situación en que se encuentran y en la que ellos podrían encontrarse en un futuro como profesionales.

(cc) EY-NC-ND 2017, Universitat Politècnica de València

Congreso IN-RED (2017) 
Cabe no olvidar, no obstante, que el empleo de esta técnica también persenta ciertas dificultades que deben ser considerados por el profesor

- Requiere cierto conocimiento de los alumnos, para poder reforzar, aquellas intervenciones menos fluidas.

- Hay que crear y aproximarse a la situación que se simula, y dadas las limitaciones del aula facilitar al máximo la atmósfera y el cambio de rol. Por ejemplo, disponiendo las sillas y mesas de modo adecuado a la entrevista, haciendo a los alumnos investigadores salir del aula y entrar como investigadores, etc.

- Es necesaria la total implicación del docente, revisando la actuación de los grupos, y evitando que se excedan en la compostura para no afectar a aulas cercanas o perder la perspectiva del tema tratado.

- Asimismo, hay que contar que en algunos casos, cuando se plantea este tipo de práctica, hay alumnos que lo ven como algo superfluo y otros como un obstáculo. Hay que lidiar con ambos y defenderla con entusiasmo y convencimiento.

\section{Conclusiones}

En la asignatura cuatrimestral de segundo curso del Grado de Ingeniero Informático de la ETSIINF, Deontología y Profesionalismo, se aplicaron las técnicas de simulación y juegos de rol a través de estas la actividad "FUGA DE DATOS EN EL HOSPITAL DE FUENLABRADA" para la realización de una de las prácticas de la asignatura.

Estas técnicas permiten de forma directa el desarrollo de la competencia CT04. La competencia innovación, creatividad y emprendimiento.

Aprovechando el uso de esta versátil actividad, se introducen conceptos relacionados con el cumplimiento de la legalidad y la actuación ética, propios de la asignatura.

La actividad permite, por tanto, trabajar en la competencia con elementos ligados a su futura profesión como ingenieros en informática que no son abordados en otras asignaturas. Cabe destacar que dicha actividad se lleva a cabo de una forma amena, interiorizada y compartida, lo cual consideramos que resulta de gran ayuda para el desarrollo de otra competencia.

Para los alumnos, una vez realizada la actividad, la sensación fue muy positiva y surgieron deseos de volver a repetir este tipo de actividades.

\section{Referencias}

García Magna, D., Castillo Rodríguez, C., Rios Moyano, S., Cristofol Rodríguez, C., Carrasco Santos, M., Rodríguez Mérida, R. M., ... \& Pastor García, I. (2011). La interdisciplinariedad en la educación superior: propuesta de una guía para el diseño de juegos de rol. Teoría de la Educación,12(1)27.

Jiménez Martín, S. y Moncholi Chaparro, M.A. (2009). El entrenamiento en técnicas creativas en el Espacio Europeo de Educación Superior. Actas del I Congreso Internacional Latina de Comunicación Social (pp. 1-12) Universidad de La Laguna. http://www.revistalatinacs.org/09/Sociedad/actas/12silvia.pdf 
Simulación y Juegos de rol como herramienta de innovación en la asignatura Deontología y

Profesionalismo

Perea Acevedo, A. J. (2008). Los juegos de rol como estrategia para la formación ética de niños y jóvenes: razones y oportunidades desde la noción foucaultiana de libertad. Infancias Imágenes, 7(1), 38-42.

Ruben, B.D. (1999). Simulations, Games, and Experience Based Learning: The Quest for a New Paradigm for Teaching and Learning. Simulation and Gaming. 30 (4), 498-505. 